\title{
Validation of an FFQ to assess antioxidant intake in overweight postmenopausal women
}

\author{
Meng Yang ${ }^{1}$, Ying Wang ${ }^{1}$, Catherine G Davis ${ }^{1}$, Sang Gil Lee ${ }^{1}$, Maria Luz Fernandez ${ }^{1}$, \\ Sung I Koo', Eunyoung $\mathrm{Cho}^{2}$ and Ock K Chun ${ }^{1, *}$ \\ 'Department of Nutritional Sciences, University of Connecticut, 3624 Horsebarn Road Extension Unit 4017, \\ Storrs, CT 06269-4017, USA: ${ }^{2}$ Channing Division of Network Medicine, Department of Medicine, Brigham and \\ Women's Hospital and Harvard Medical School, Boston, MA, USA
}

Submitted 19 November 2012: Final revision received 1 April 2013: Accepted 9 May 2013: First published online 26 June 2013

\begin{abstract}
Objective: To validate an FFQ to assess antioxidant intake in overweight postmenopausal women.

Design: A seventy-four-item antioxidant 1-month FFQ was developed based on major antioxidant sources in the American diet. Forty overweight postmenopausal women participated in a 9-month observational study and completed four sets of FFQ and $7 \mathrm{~d}$ food record ( $7 \mathrm{dFR}$ ) every 3 months. Twelve-hour fasting blood was collected for plasma antioxidant measurement at the first visit.

Setting: Connecticut, USA.

Subjects: Forty overweight postmenopausal women.

Results: Spearman correlation coefficients of 1-month antioxidant intake estimated from the first set of FFQ and 7dFR ranged from $0 \cdot 34$ to $0 \cdot 87$, except for $\gamma$-tocopherol. The proportion of participants categorized into the extremely opposite tertiles averaged $7 \%$. Significant correlations were observed for diet-plasma vitamin C, $\alpha$-tocopherol and carotenoids $(P<0 \cdot 05)$. No time effect was observed on the dietary antioxidant intakes estimated from four $7 \mathrm{dFR}$ and four FFQ. Dietary antioxidants estimated from averaged four 7dFR showed moderate to high correlation with those estimated from averaged four FFQ and from each FFQ collected every 3 months. Bland-Altman plots did not show any systematic bias. Averaged misclassifications were below $10 \%$ between these two instruments.

Conclusions: These findings attested a reasonable validity and a good acceptance of this 1-month FFQ in assessing both short-term and long-term diverse antioxidant intakes in these overweight postmenopausal women. The use of this FFQ in associating antioxidant intake with disease risk needs further investigation.
\end{abstract}

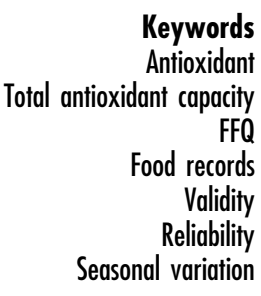

The antioxidative properties of multiple bioactive compounds have been proposed to partially contribute to the protective effects of vegetables and fruits on chronic diseases $^{(1,2)}$. These compounds include various antioxidants including ascorbic acid, tocopherols, carotenoids, and polyphenols such as simple phenolics, flavonoids and proanthocyanidins. In epidemiological or clinical investigations, several studies have reported inverse associations between dietary antioxidants and incidences of CVD or cancer $^{(3-6)}$, while others did not confirm these findings ${ }^{(7-9)}$. Therefore, clarification on the health implications of dietary antioxidants needs to be continuously explored, which, to a large extent, relies upon the quality of dietary exposure assessment $^{(10)}$.

Despite the potential importance of various antioxidants, there is a paucity of development and validation studies, especially of dietary flavonoids, which probably stems from methodological limitations imposed by a lack of flavonoid composition data for foods. The self-administered FFQ is the primary method of dietary assessment used in epidemiological studies ${ }^{(11)}$, owing to its capability of capturing a long-term dietary behaviour, low participant burden and ease in data processing. A new FFQ was developed to assess a comprehensive antioxidant intake profile containing vitamin $\mathrm{C}$, vitamin $\mathrm{E}$, carotenoids, flavonoids and proanthocyanidins, as well as total antioxidant capacity (TAC), an integrated measure of the 'quenching abilities' of antioxidants $^{(12)}$. This FFQ provided a reasonable validity in a sample of American college students ${ }^{(12)}$. Nevertheless, since this FFQ was initially created to assess short-term antioxidant intake status (1 month), whether it could be applied in other populations and whether it could be extended to measure usual antioxidant intake needed further investigation. The aims of the present study therefore were to evaluate the validity of the newly developed FFQ in overweight postmenopausal women and to extend 
its feasibility in assessing long-term antioxidant intake with respect to this target group.

\section{Materials and methods}

\section{Participants and study design}

A 9-month observational study was conducted in 40 70-year-old, overweight/obese $\left(\mathrm{BMI}=25 \cdot 0-39 \cdot 9 \mathrm{~kg} / \mathrm{m}^{2}\right)$, non-smoking postmenopausal women. Volunteers were identified as potential subjects through a telephone interview and were immediately invited to the Clinical Research Center located at the University of Connecticut Health Center in Farmington, Connecticut, USA. On the initial visit, after written informed consent was obtained, anthropometric parameters (height, weight) and blood pressure were measured by one nurse in the University of Connecticut Health Center to determine the eligibility of participants. Height was measured by having the participants stand against a wall ruler without shoes. Weight was measured using the scale provided to the participants. Two measurements of blood pressures, $45 \mathrm{~s}$ apart, were taken on the participants' left arms. Data recorded for each measurement included systolic and diastolic blood pressure and the time of day the reading was taken. An interview performed by the same nurse was followed regarding the participants' medical, dietary, smoking and alcohol consumption histories. Exclusion criteria included: (i) having any history of chronic disease such as diabetes mellitus, CVD, arthritis (except for osteoarthritis), kidney disease, currently being treated for cancer (i.e. chemotherapy, radiation therapy) and any history of malnutrition or digestion problems; (ii) taking any anti-inflammatory medicines; (iii) following slimming diets; and (iv) consuming alcohol exceeding 2 drinks/d or a total of 12 drinks/week. This population was specifically chosen because obesity and postmenopausal status serve as significant risk factors of chronic inflammation and $\mathrm{CVD}^{(13,14)}$. The data from this population will be further used for FFQ application. If the participants were eligible, a $12 \mathrm{~h}$ fasting venous blood sample was taken. A dietitian instructed the participants on how to record a $7 \mathrm{~d}$ food record ( $7 \mathrm{dFR}$ ). At the second visit seven days after the initial screening, the eligible participants submitted the $7 \mathrm{dFR}$ and completed the newly developed FFQ (FFQ1). After the second visit, participants were requested to complete one set of FFQ along with a $7 \mathrm{dFR}$ in each season and mail them back to the research team. In total, four sets of FFQ and 7dFR were collected and numbered 1 to 4 chronologically. Among forty-one eligible participants, forty completed the second visit. All but five volunteers (88\%) finished and submitted three other sets of FFQ and 7dFR during 9 months.

\section{Dietary assessment by $F F Q$}

The FFQ is a questionnaire that integrates nine food groups into seventy-four questions, including four on dietary supplements, aiming to estimate a comprehensive dietary antioxidant profile (vitamin $\mathrm{C}$, vitamin $\mathrm{E}$, carotenoids, flavonoids, proanthocyanidins) along with TAC values. The food list in the FFQ was determined based on the ranking of TAC scores of mostly consumed food items in the American $\operatorname{diet}^{(15)}$, using food consumption data of 8809 US adults in the National Health and Nutrition Examination Survey (NHANES) 1999-2002 $2^{(16,17)}$. The US Department of Agriculture's flavonoid and proanthocyanidin data sets ${ }^{(18-20)}$ and the antioxidant capacity data set of forty-three major antioxidant nutrients ${ }^{(21)}$ were used to calculate TAC scores. Antioxidant capacity of each antioxidant from the food items was calculated by multiplying daily consumption of each antioxidant by its corresponding antioxidant capacity. TAC score from the specific food consumed daily was assessed by summing individual antioxidant capacities. The top food items contributing most to TAC in this food list were selected to cover at least $83 \%$ of the cumulative TAC and were translated into seventy questions. The final FFQ contained eight food groups without specific information such as preparations (fifteen vegetables and vegetable products, eighteen fruits, twenty-one beverages, two breads and cereals, six dairy and eggs, four oils and seasonings, two sweets and desserts, and two others such as nuts or seeds). Since the TAC scores of vitamin $\mathrm{C}, \alpha$-tocopherol and $\beta$-carotene from dietary supplements contributed to almost $25 \%$ of the TAC from diet and supplements in Americans according to the previous study ${ }^{(15)}$, vitamin $\mathrm{C}, \alpha$-tocopherol, $\beta$-carotene and multivitamins were included in the four dietary supplement questions with dosage information nested. Because limited information is available on supplementary flavonoid composition, and the flavonoid intake from supplements was documented to be less than $2 \%$ in US adults ${ }^{(15,22)}$, flavonoid intake from supplements was not included. Food frequency was coded as daily, weekly and monthly, and from 0 to 7 occasions including none, 1 time/month, $<1$ time/week, 1-2 times/week, 3-4 times/week, 5-6 times/ week, 1 time/d and $>1$ time/d. The FFQ was intended to cover the previous 1-month consumption of food and supplements. Portion sizes were estimated using three different scales (small, medium and large); small serving was half of the medium serving, while large serving equalled one-andhalf of the medium serving. Photographic figures for medium serving size were included to illustrate the portion size.

To calculate antioxidant intake from the FFQ, a 'unit' antioxidant database based on a 'medium' serving size was created by combining the dietary nutrient profile for individual food items from the Nutrition Data System for Research (NDSR) release 2010 (University of Minnesota, Minneapolis, MN, USA) with the Flavonoid/Proanthocyanidin Provisional Table developed by the Nutrition Coordinating Center (NCC). This NCC Provisional Table provided a way for NDSR users to link the US Department of Agriculture data with NDSR data via NDSR food identification codes ${ }^{(23)}$. Frequency from the FFQ was converted proportionally to daily units. Consequently, daily antioxidant intake from 
food was obtained by multiplying the 'unit' antioxidant database by the frequency and the factor of the reported portion size relative to a 'medium' serving. Vitamin $C, \alpha$-tocopherol and $\beta$-carotene intakes from dietary supplements were determined from the addition of single-nutrient supplements and multivitamin use according to the default dietary supplements database in NHANES 2007-2008 ${ }^{(24)}$. The mean daily dose of multivitamins was calculated by the frequency of intake. Individual antioxidant capacity for a specific food item or dietary supplement was obtained through multiplying the daily antioxidant intake by the corresponding antioxidant capacity. TAC of each food item or dietary supplement was calculated by summing the individual antioxdiant capacities in the foods or supplements. TAC from the whole diet was calculated by summing all TAC scores from individual food or dietary supplements ${ }^{(15)}$.

\section{Dietary assessment by food record}

The study dietitian trained the participants to complete a $7 \mathrm{dFR}$ by including all foods, beverages and dietary supplements consumed during the seven consecutive days and reviewed the records to check for errors or omissions every day. Dietary intake data were collected and analysed by using NDSR and the Flavonoid/Proanthocyanidin Provisional Table. Dietary supplement data were estimated through NDSR updated with an NCC enhanced version of the NHANES Dietary Supplement Database 2007-2008 ${ }^{(23)}$. TAC from diet and TAC from supplements were obtained through multiplying antioxidant profiles by antioxidant capacities.

\section{Measurements of plasma antioxidants, total antioxidant capacity and lipids}

A $12 \mathrm{~h}$ fasting blood sample for plasma antioxidant analysis was collected in vacutainers containing heparin sodium or EDTA at the initial visit. Samples were centrifuged immediately at $3000 \mathrm{~g}$ for $10 \mathrm{~min}$ at $4{ }^{\circ} \mathrm{C}$. Plasma was separated and stored at $-80{ }^{\circ} \mathrm{C}$ until further measurements. Plasma vitamin $\mathrm{C}$ was measured on deproteinized plasma by HPLC with a UV detector as described by Ross ${ }^{(25)}$. In order to preserve vitamin $\mathrm{C}$, an aliquot of plasma was deproteinized with $10 \%(\mathrm{w} / \mathrm{v})$ perchloric acid. This sample was then centrifuged $\left(15000 \mathrm{~g}, 5 \mathrm{~min}, 4^{\circ} \mathrm{C}\right)$ and the supernatant was kept at $-80^{\circ} \mathrm{C}$ until analysis. Plasma $\alpha$-tocopherol and $\gamma$-tocopherol were analysed using HPLC $^{(26)}$. The slightly modified method described by Karppi et al. ${ }^{(27)}$ was used for carotenoid analyses. Plasma TAC was determined by the ABTS assay developed by van den Berg et $a l^{(28)}$ and modified by Floegel et ll $^{(29)}$. Lipid profiles including total cholesterol and TAG were measured with the Cobas C111 analyser (Roche Diagnostics, Indianapolis, IN, USA).

\section{Statistical analysis}

All statistical analyses were carried out using the SAS statistical software package version $9 \cdot 2$. Descriptive statistics were computed to describe sociodemographic characteristics, averaged daily antioxidant intake estimated from the first set of 7dFR and FFQ, and plasma antioxidant and TAC levels. For validity testing, Spearman rank correlation coefficients were calculated between the dietary nutrients estimated from 7dFR1 and FFQ1 and between the corresponding dietary and plasma nutrients after adjusting for age, BMI, ethnicity and (except for vitamin C) plasma cholesterol and TAG concentrations. This was also performed for antioxidant intakes estimated from FFQ1 and FFQ4 for testing reliability. Bland-Altman plots were generated to assess the agreement between the first set of $7 \mathrm{dFR}$ and $\mathrm{FFQ}^{(30,31)}$. Repeated ANOVA was performed to compare antioxidant intakes or the respective major food sources estimated from four $7 \mathrm{dFR}$ or four FFQ for examining seasonal variations of dietary antioxidants. To test whether this FFQ was able to assess long-term antioxidant intake, Spearman rank correlations were calculated between antioxidant intakes estimated from averaged four $7 \mathrm{dFR}$, those from averaged four FFQ and those obtained from four individual FFQ, respectively. In addition, cross-classification was assessed by calculating the percentages of respondents classified into the same third or the adjacent third of antioxidant intake by $7 \mathrm{dFR} 1$ and FFQ1, and by averaged four 7dFR, averaged four FFQ and four individual FFQ. Misclassification was reported as the percentage of respondents categorized into the extreme opposite tertiles. Significance was set at a value of $P<0 \cdot 05$ for two-sided testing.

\section{Results}

The forty participants who completed the second visit for the FFQ validity test were predominantly non-Hispanic white, with an average age of 58 years and a BMI of $30.0 \mathrm{~kg} / \mathrm{m}^{2}$ (Table 1). Sixteen participants reported taking a daily multivitamin. Three took a daily vitamin $\mathrm{C}$ only supplement, two took a vitamin E only supplement and none of them took a $\beta$-carotene only supplement.

Daily consumption of vitamin E, vitamin C, carotenoids, flavonoids and proanthocyanidins estimated from FFQ1 accounted for $50 \cdot 1 \%$ (88.9\% including vitamin E supplements), $93 \cdot 5 \%$ (106.6\% including vitamin C supplements), $82 \cdot 8 \%, 77 \cdot 2 \%$ and $89 \cdot 8 \%$, respectively, of those from average 7dFR1. TAC estimated by FFQ1 was approximately $80 \%$ of the value calculated from $7 \mathrm{dFR} 1$ (Table 2). There was a moderately to highly positive correlation between dietary intake estimated from the first set of FFQ and 7dFR for individual antioxidants and TAC, except for $\gamma$-tocopherol ( $r=0.34$ to $0 \cdot 87, P<0 \cdot 05$; Table 2 ). The proportion of participants categorized in the opposite tertiles averaged $7 \%$ (Table 2). Significant Spearman correlations were observed between dietary vitamin $\mathrm{C}$ and carotenoids ( $\beta$-carotene, $\beta$-cryptoxanthin, lutein + zeaxanthin, lycopene) estimated from FFQ1 and their corresponding plasma levels, in the 
range of 0.35 to $0.50(P<0.05)$ after adjusting for relevant covariates. $\alpha$-Tocopherol intake from diet only did not show any correlation with plasma $\alpha$-tocopherol level; however, including supplementary $\alpha$-tocopherol greatly increased the

Table 1 Sociodemographic characteristics, antioxidant supplement use and plasma antioxidant concentrations of overweight and obese postmenopausal women who completed the first set of FFQ and $7 \mathrm{dFR}(n$ 40)

\begin{tabular}{|c|c|c|}
\hline Characteristic & Mean or $n$ & SD \\
\hline Age (years) & $58 \cdot 2$ & $6 \cdot 1$ \\
\hline BMI $\left(\mathrm{kg} / \mathrm{m}^{2}\right)$ & $30 \cdot 3$ & $3 \cdot 1$ \\
\hline \multicolumn{3}{|l|}{ Ethnicity $(n)$} \\
\hline Non Hispanic white & 36 & - \\
\hline Non-Hispanic black & 2 & - \\
\hline Hispanic & 0 & - \\
\hline Others & 2 & - \\
\hline \multicolumn{3}{|l|}{ Supplement use $(n)^{*}$} \\
\hline Multivitamins & 16 & - \\
\hline Vitamin C only & 3 & - \\
\hline Vitamin E only & 2 & - \\
\hline$\beta$-Carotene only & 0 & - \\
\hline None & 22 & - \\
\hline \multicolumn{3}{|l|}{ Plasma antioxidant/TAC levels } \\
\hline Vitamin C $(\mu \mathrm{mol} / \mathrm{l})$ & $45 \cdot 85$ & $15 \cdot 86$ \\
\hline$\alpha$-Tocopherol $(\mu \mathrm{mol} / \mathrm{l})$ & $32 \cdot 25$ & 9.03 \\
\hline$\gamma$-Tocopherol $(\mu \mathrm{mol} / \mathrm{l})$ & $2 \cdot 43$ & $1 \cdot 31$ \\
\hline$\alpha$-Carotene $(\mu \mathrm{mol} / \mathrm{l})$ & $0 \cdot 10$ & $0 \cdot 12$ \\
\hline$\beta$-Carotene $(\mu \mathrm{mol} / \mathrm{l})$ & $0 \cdot 19$ & $0 \cdot 17$ \\
\hline$\beta$-Cryptoxanthin ( $\mu \mathrm{mol} / \mathrm{l})$ & 0.26 & $0 \cdot 17$ \\
\hline Lutein + zeaxanthin $(\mu \mathrm{mol} / \mathrm{l})$ & $1 \cdot 64$ & $9 \cdot 21$ \\
\hline Lycopene $(\mu \mathrm{mol} / \mathrm{l})$ & $0 \cdot 11$ & 0.05 \\
\hline TAC (mg VCE/l)t & $302 \cdot 3$ & $25 \cdot 2$ \\
\hline
\end{tabular}

7dFR, 7d food record; TAC, total antioxidant capacity; VCE, vitamin C equivalent.

*Supplement use was estimated based on the first 7dFR.

tPlasma TAC was measured by the ABTS assay and expressed as mg VCE/l. correlation $(r=0 \cdot 15$ and $0 \cdot 74$, respectively). Neither TAC from diet nor TAC from diet and supplements was associated with plasma TAC level (Table 3). Bland-Altman plots exemplified by dietary vitamin E, vitamin C, carotenoids, flavonoids, proanthocyanindins and TAC showed that the plotted points remained predominantly within the 95\% limits of agreement, suggesting no systematic bias in measurement using the FFQ compared with 7dFR (Fig. 1).

Dietary antioxidants estimated from four $7 \mathrm{dFR}$ and four FFQ collected every season are shown in Fig. 2. No time effect was observed for antioxidant intakes over 9 months. However, investigations on supplement intake collected by four FFQ showed that neither frequency nor amount of dietary supplements was consistent in these overweight postmenopausal women (data not shown). Therefore, subsequent analyses on long-term antioxidant intake did not include dietary supplements from each FFQ.

Correlations of dietary antioxidant estimates from averaged four sets of 7dFR with averaged four FFQ, FFQ1 and FFQ4 collected during 9 months are shown in Table 4. Compared with antioxidant intake estimated from averaged four 7dFR, significant Spearman correlations to examine validity were found to fall within the range from 0.52 to 0.91 for averaged four FFQ except for $\gamma$-tocopherol and flavones, from 0.38 to 0.86 for FFQ1 except for $\gamma$-tocopherol and lutein + zeaxanthin, and from 0.38 to 0.78 for FFQ4 except for $\boldsymbol{\gamma}$-tocopherol, lutein + zeaxanthin and flavones $(P<0 \cdot 05)$. On the basis of all the antioxidants among averaged four FFQ, FFQ1 and FFQ 4 compared with averaged four $7 \mathrm{dFR}, 4 \%, 4 \%$

Table 2 Averaged daily antioxidant intakes and validity test (Spearman correlation coefficients and misclassification percentage) between the first set of 7dFR and FFQ collected from overweight and obese postmenopausal women ( $n$ 40)

\begin{tabular}{|c|c|c|c|c|c|c|c|c|c|}
\hline \multirow[b]{3}{*}{ Nutrient } & \multicolumn{6}{|c|}{ Daily intake } & \multicolumn{3}{|c|}{ Validity test } \\
\hline & \multicolumn{3}{|c|}{$7 \mathrm{dFR}$} & \multicolumn{3}{|c|}{ FFQ1 } & \multicolumn{2}{|c|}{ Spearman correlation } & \multirow[b]{2}{*}{$\%$ Mis-C } \\
\hline & Mean & SD & Median & Mean & SD & Median & $r$ & $P$ value & \\
\hline \multicolumn{10}{|l|}{ Dietary antioxidants } \\
\hline Vitamin $E(\mathrm{mg} / \mathrm{d})$ & $24 \cdot 3$ & $18 \cdot 3$ & $22 \cdot 0$ & $12 \cdot 1$ & $9 \cdot 7$ & $10 \cdot 6$ & 0.34 & $<0.05$ & 10 \\
\hline$\alpha$-Tocopherol (mg/d) & $9 \cdot 1$ & $4 \cdot 2$ & $8 \cdot 1$ & $4 \cdot 5$ & $2 \cdot 9$ & $3 \cdot 8$ & 0.48 & $<0.01$ & $2 \cdot 5$ \\
\hline$\gamma$-Tocopherol (mg/d) & $10 \cdot 4$ & $4 \cdot 1$ & $9 \cdot 9$ & $5 \cdot 7$ & $4 \cdot 8$ & $4 \cdot 7$ & $0 \cdot 13$ & 0.427 & 25 \\
\hline Vitamin C (mg/d) & $98 \cdot 3$ & 48 & $96 \cdot 3$ & 91.9 & $59 \cdot 2$ & $84 \cdot 6$ & 0.59 & $<0.0001$ & $2 \cdot 5$ \\
\hline Carotenoids $(\mu \mathrm{g} / \mathrm{d})$ & 13680 & 8220 & 12009 & 11331 & 7278 & 9432 & 0.63 & $<0.0001$ & $12 \cdot 5$ \\
\hline$\alpha$-Carotene $(\mu \mathrm{g} / \mathrm{d})$ & 515 & 570 & 337 & 339 & 319 & 168 & 0.48 & $<0.01$ & $7 \cdot 5$ \\
\hline$\beta$-Carotene $(\mu \mathrm{g} / \mathrm{d})$ & 4162 & 2709 & 3785 & 2288 & 1510 & 1984 & 0.57 & $<0.001$ & $7 \cdot 5$ \\
\hline$\beta$-Cryptoxanthin $(\mu \mathrm{g} / \mathrm{d})$ & 162 & 269 & 111 & 70 & 55 & 60 & 0.38 & $<0.05$ & 10 \\
\hline Lutein + zeaxanthin $(\mu \mathrm{g} / \mathrm{d})$ & 3013 & 3075 & 2287 & 2341 & 2436 & 1299 & 0.52 & $<0.001$ & 5 \\
\hline Lycopene $(\mu \mathrm{g} / \mathrm{d})$ & 5829 & 4973 & 4809 & 6293 & 5418 & 4719 & 0.45 & $<0.01$ & $12 \cdot 5$ \\
\hline Flavonoids (mg/d) & $238 \cdot 6$ & $236 \cdot 4$ & $161 \cdot 3$ & $184 \cdot 3$ & $155 \cdot 4$ & $153 \cdot 6$ & 0.76 & $<0.0001$ & 0 \\
\hline Isoflavones (mg/d) & 3.5 & $6 \cdot 8$ & $1 \cdot 0$ & $2 \cdot 8$ & $10 \cdot 6$ & 0.3 & 0.40 & $<0.05$ & $7 \cdot 5$ \\
\hline Anthocyanins $(\mathrm{mg} / \mathrm{d})$ & $67 \cdot 7$ & 57 & $58 \cdot 9$ & $68 \cdot 9$ & $87 \cdot 4$ & $35 \cdot 7$ & 0.50 & $<0.001$ & 5 \\
\hline Flavan-3-ols (mg/d) & $134 \cdot 1$ & $210 \cdot 2$ & $60 \cdot 7$ & $73 \cdot 1$ & $72 \cdot 3$ & $46 \cdot 3$ & 0.69 & $<0.0001$ & $2 \cdot 5$ \\
\hline Proanthocyanidins (mg/d) & $40 \cdot 6$ & $37 \cdot 2$ & $30 \cdot 8$ & $36 \cdot 5$ & $25 \cdot 7$ & $31 \cdot 4$ & 0.68 & $<0.0001$ & 5 \\
\hline Flavanones $(\mathrm{mg} / \mathrm{d})$ & $10 \cdot 7$ & $11 \cdot 9$ & $6 \cdot 6$ & $18 \cdot 2$ & $23 \cdot 0$ & $8 \cdot 8$ & 0.42 & $<0.01$ & $7 \cdot 5$ \\
\hline Flavones $(\mathrm{mg} / \mathrm{d})$ & $2 \cdot 2$ & $2 \cdot 3$ & $1 \cdot 8$ & $1 \cdot 4$ & $1 \cdot 3$ & $1 \cdot 0$ & 0.78 & $<0.0001$ & 0 \\
\hline Flavonols (mg/d) & 20.5 & $15 \cdot 1$ & $15 \cdot 5$ & $20 \cdot 0$ & $14 \cdot 7$ & $15 \cdot 9$ & 0.46 & $<0.01$ & 10 \\
\hline $\mathrm{TAC}(\mathrm{mg} \mathrm{VCE} / \mathrm{d})$ & $686 \cdot 6$ & 578 & $541 \cdot 4$ & $550 \cdot 4$ & $406 \cdot 8$ & $447 \cdot 2$ & 0.76 & $<0.0001$ & $2 \cdot 5$ \\
\hline \multicolumn{10}{|l|}{ Dietary + supplement antioxidants } \\
\hline$\alpha$-Tocopherol $(\mathrm{mg} / \mathrm{d})$ & $60 \cdot 5$ & $130 \cdot 3$ & $11 \cdot 1$ & $59 \cdot 7$ & $133 \cdot 9$ & $7 \cdot 8$ & 0.87 & $<0.0001$ & $2 \cdot 5$ \\
\hline Vitamin $C(\mathrm{mg} / \mathrm{d})$ & $185 \cdot 3$ & $187 \cdot 4$ & $120 \cdot 3$ & $197 \cdot 5$ & $252 \cdot 6$ & $131 \cdot 8$ & 0.74 & $<0.0001$ & $7 \cdot 5$ \\
\hline
\end{tabular}

7dFR, $7 \mathrm{~d}$ food record; \% Mis-C, percentage of misclassification; $r$, correlation coefficient; TAC, total antioxidant capacity; VCE, vitamin C equivalent. ${ }^{*}$ Percentage of respondents categorized into the extreme opposite tertile. 
Table 3 Spearman rank correlations between dietary antioxidants estimated from the first set of FFQ and 7dFR and corresponding plasma antioxidant levels in overweight and obese postmenopausal women who completed the first set of FFQ and 7d FR $(n 40)^{*}$

\begin{tabular}{|c|c|c|c|c|}
\hline \multirow[b]{3}{*}{ Plasma $v$. diet } & \multicolumn{2}{|c|}{ FFQ1 } & \multicolumn{2}{|c|}{$7 \mathrm{dFR} 1$} \\
\hline & \multicolumn{2}{|c|}{ Spearman correlation } & \multicolumn{2}{|c|}{ Spearman correlation } \\
\hline & $r$ & $P$ value & $r$ & $P$ value \\
\hline$\alpha$-Tocopherolt & $0 \cdot 15$ & 0.384 & 0.32 & 0.061 \\
\hline$\alpha$-Tocopherol (diet + supplements) $\ddagger$ & $0 \cdot 74$ & $<0.001$ & $0 \cdot 76$ & $<0.001$ \\
\hline$\gamma$-Tocopherol & 0.06 & $0 \cdot 718$ & $0 \cdot 11$ & 0.521 \\
\hline Vitamin C & $0 \cdot 41$ & $<0.05$ & $0 \cdot 19$ & 0.305 \\
\hline Vitamin C (diet + supplements) & $0 \cdot 33$ & 0.068 & $0 \cdot 18$ & 0.319 \\
\hline$\alpha$-Carotene & $0 \cdot 24$ & $0 \cdot 174$ & $0 \cdot 39$ & $<0.05$ \\
\hline$\beta$-Carotene & 0.44 & $<0.01$ & $0 \cdot 34$ & $<0.05$ \\
\hline$\beta$-Cryptoxanthin & $0 \cdot 50$ & $<0.01$ & -0.05 & $0 \cdot 770$ \\
\hline Lutein + zeaxanthin & 0.50 & $<0.01$ & 0.47 & $<0.01$ \\
\hline Lycopene & $0 \cdot 35$ & $<0.05$ & $0 \cdot 30$ & 0.078 \\
\hline TAC\$ & 0.25 & 0.162 & $0 \cdot 12$ & 0.495 \\
\hline
\end{tabular}

$7 \mathrm{dFR}, 7 \mathrm{~d}$ food record; $r$, correlation coefficient; TAC, total antioxidant capacity.

*Models were adjusted for age, gender, ethnicity, BMl and (except for vitamin C) plasma cholesterol and plasma TAG concentrations, and supplement use. tSpearman correlations were calculated between dietary antioxidants and corresponding plasma concentrations.

$\ddagger$ Spearman correlations were calculated between dietary and supplementary antioxidants and corresponding plasma concentrations.

$\S$ Models were adjusted for age, gender, ethnicity, BMI, plasma uric acid concentration and supplement use.
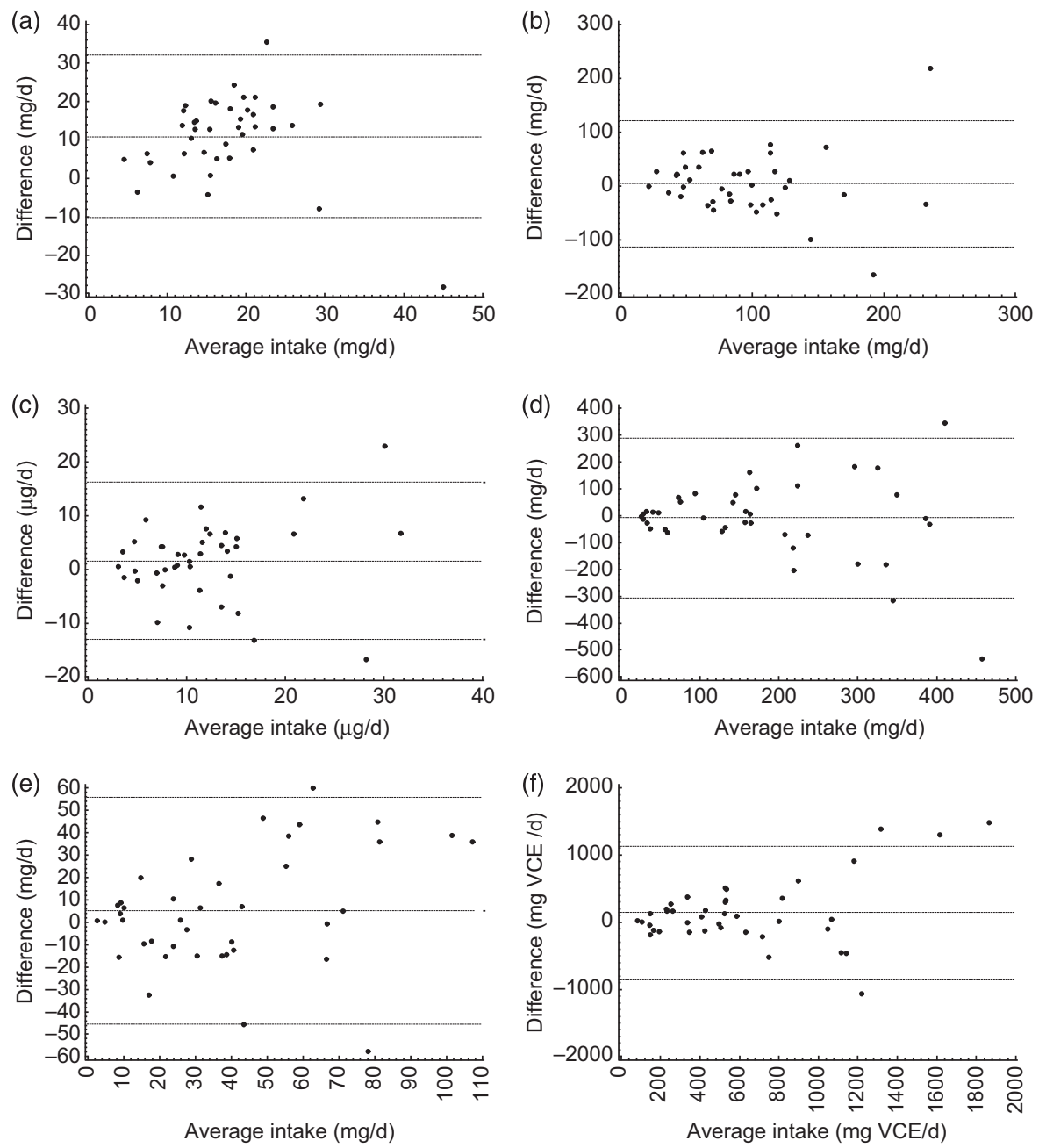

Fig. 1 Bland-Altman plots comparing the dietary intake of (a) vitamin E, (b) vitamin C, (c) total carotenoids, (d) flavonoids, (e) proanthocyanidins and ( $f$ ) total antioxidant capacity measured using the first set of $7 \mathrm{~d}$ food records and $F F Q$ collected from overweight and obese postmenopausal women ( $n$ 40). The middle line represents the mean difference between the two assessment methods; the upper and lower lines are the $95 \%$ limits of agreement. VCE, vitamin $\mathrm{C}$ equivalent 


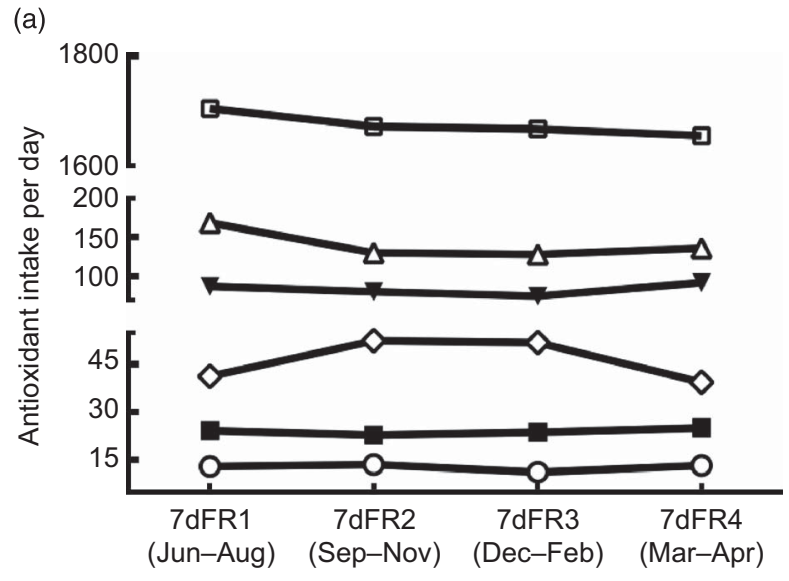

Daily antioxidant intake

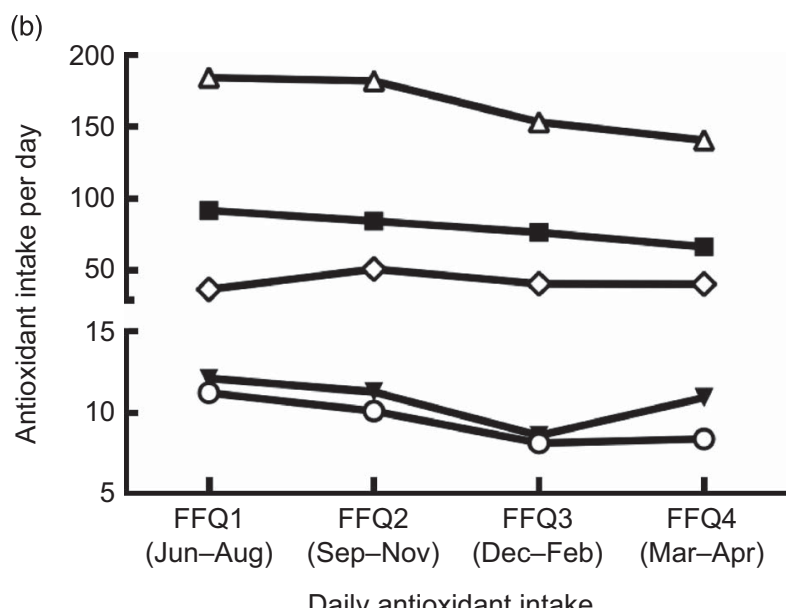

Fig. 2 Comparisons among dietary antioxidant intakes (- $\square$, energy $(\mathrm{kcal} / \mathrm{d} ; 1 \mathrm{kcal}=4.184 \mathrm{~kJ}) ;-\nabla-$, vitamin $\mathrm{E}(\mathrm{mg} / \mathrm{d})$; - -, vitamin C $(\mathrm{mg} / \mathrm{d}) ;-\bigcirc-$, carotenoids $(\mu \mathrm{g} / \mathrm{d}) ;-\triangle-$, flavonoids (mg/d); - $\diamond-$, proanthrocyanidins $(\mathrm{mg} / \mathrm{d})$ ) estimated from (a) four sets of $7 \mathrm{~d}$ food records (7dFR) and (b) four sets of FFQ collected every 3 months in overweight and obese postmenopausal women (n 35). There was no time effect on antioxidant intake over 9 months

and $9 \%$ on average were misclassified into the opposite tertiles, respectively (Table 4). Correlation coefficients to estimate test-retest reliability between FFQ1 and FFQ4 collected 9 months apart were found to be higher than 0.5 for individual antioxidants and TAC (Table 5).

\section{Discussion}

The present study demonstrated the reasonable validity of an FFQ to comprehensively assess short-term antioxidant intake in overweight postmenopausal women. It also validated this FFQ in estimating a longer-term antioxidant intake through justifying the seasonal variability of antioxidant intake. This extension improved the FFQ applicability in epidemiological and clinical settings. Different statistical methods were used for testing FFQ validity, including
Spearman rank correlation, cross-classification and BlandAltman plots. Biochemical surrogates were added as a comparison measure, as the measurement errors from biochemical markers and the FFQ are independent ${ }^{(32)}$.

Daily intakes of vitamin C, several carotenoids and flavonoids, proanthocyanidins and TAC estimated from the $7 \mathrm{dFR}$ and the FFQ were generally comparable with a difference of $10 \%$ to $30 \%$. However, daily intakes of vitamin E ( $\alpha$-tocopherol and $\gamma$-tocopherol), certain carotenoids ( $\alpha$-carotene, $\beta$-carotene and $\beta$-cryptoxanthin) and flavonoids (flavan-3-ols, flavanones and flavones) from the FFQ were considerably lower than those from $7 \mathrm{dFR}$. These results, to some extent, emphasize that the FFQ could not be used for evaluating the absolute intake quantitatively. However, the FFQ is usually used to rank individuals in a target population according to dietary nutrients rather than to quantify estimates. Among these overweight postmenopausal women, we found moderate to high correlations of short-term antioxidant intake between the first set of FFQ and 7dFR. These validation data were generally comparable to those reported by previous studies using food records as a 'gold standard' reference method. Correlations observed between these two assessment tools were within the ranges noted by other investigators, from 0.27 to $0 \cdot 71$ for vitamin $C^{(33-37)}$, from 0.33 to 0.75 for vitamin $\mathrm{E}^{(38-40)}$ and from 0.14 to 0.38 for total carotenoids and respective subclasses ${ }^{(41,42)}$. Additionally, since the correlation analysis for testing validity has been questioned for its failure in measuring agreement ${ }^{(30,43)}$, Bland-Altman plots and cross-classification were used in the present study to bridge this gap ${ }^{(44)}$. Bland-Altman plots did not show any systematic bias. Cross-classification indicated an acceptable low misclassification percentage of antioxidant groups, although individual antioxidants had relatively higher non-agreements. Furthermore, positive associations were demonstrated for most antioxidants estimated from FFQ with plasma concentrations, although most correlation coefficients were less than 0.50 . Nevertheless, diet-plasma coefficients observed for $\alpha$-tocopherol and a number of carotenoid subclasses were within the range reported previously (sometimes higher) ${ }^{(32,41,42,45)}$. Of note, as indicated before ${ }^{(45)}$, plasma $\alpha$-tocopherol level was probably not a good predictor of the diet source only, while including dietary supplements significantly improved the association. The use of plasma vitamin $\mathrm{C}$ and TAC is limited and did not produce consistent correlations ${ }^{(40,46)}$, suggesting a cautious approach is needed when using these surrogate measurements of dietary estimates. To summarize, the combination of correlation coefficients and agreement measurements as well as biochemical indicators provided sufficient data to support the overall ability of this FFQ in assessing antioxidant intakes during a short-term period in these overweight postmenopausal women.

However, in epidemiological studies, the underlying principle of using an FFQ is to obtain usual dietary exposures over weeks, months or years ${ }^{(47)}$. Dietary antioxidants 
Table 4 Spearman correlation coefficients and misclassification percentage of dietary antioxidants and TAC between averaged four 7dFR, averaged four FFQ, FFQ1 and FFQ4 in overweight and obese postmenopausal women who completed four sets of 7dFR and FFQ during 9 months $(n 35)^{*}$

\begin{tabular}{|c|c|c|c|c|c|c|c|c|c|}
\hline \multirow[b]{2}{*}{ Nutrient from diet } & \multicolumn{3}{|c|}{ Ave $7 \mathrm{dFR} v$. Ave FFQ } & \multicolumn{3}{|c|}{ Ave 7dFR $v$. FFQ1 } & \multicolumn{3}{|c|}{ Ave $7 \mathrm{dFR} v$. FFQ4 } \\
\hline & $r$ & $P$ & $\%$ Mis-Ct & $r$ & $P$ & $\%$ Mis-Ct & $r$ & $P$ & $\%$ Mis-C + \\
\hline Vitamin $E(m g / d)$ & 0.56 & $<0.01$ & 3.5 & 0.54 & $<0.01$ & $7 \cdot 1$ & 0.47 & $<0.05$ & $7 \cdot 1$ \\
\hline$\alpha$-Tocopherol (mg/d) & $0 \cdot 70$ & $<0.0001$ & $3 \cdot 5$ & 0.64 & $<0.001$ & $3 \cdot 6$ & 0.72 & $<0.0001$ & $3 \cdot 6$ \\
\hline$\gamma$-Tocopherol (mg/d) & $0 \cdot 24$ & $0 \cdot 221$ & $14 \cdot 3$ & $0 \cdot 30$ & $0 \cdot 124$ & $17 \cdot 9$ & $0 \cdot 21$ & $0 \cdot 295$ & $17 \cdot 9$ \\
\hline Vitamin C $(\mathrm{mg} / \mathrm{d})$ & $0 \cdot 84$ & $<0.0001$ & 0 & 0.68 & $<0.0001$ & 0 & 0.79 & $<0.0001$ & $3 \cdot 6$ \\
\hline Carotenoids $(\mu / d)$ & 0.52 & $<0.01$ & $10 \cdot 7$ & 0.53 & $<0.01$ & $7 \cdot 1$ & $0 \cdot 44$ & $<0.05$ & $10 \cdot 7$ \\
\hline$\alpha$-Carotene $(\mu \mathrm{g} / \mathrm{d})$ & 0.56 & $<0.01$ & $3 \cdot 5$ & 0.58 & $<0.01$ & $4 \cdot 0$ & 0.63 & $<0.001$ & $21 \cdot 4$ \\
\hline$\beta$-Carotene $(\mu \mathrm{g} / \mathrm{d})$ & $0 \cdot 78$ & $<0.0001$ & 0 & $0 \cdot 75$ & $<0.0001$ & $4 \cdot 0$ & 0.47 & $<0.05$ & $10 \cdot 7$ \\
\hline$\beta$-Cryptoxanthin $(\mu \mathrm{g} / \mathrm{d})$ & $0 \cdot 70$ & $<0.0001$ & $7 \cdot 1$ & $0 \cdot 38$ & $<0.05$ & $7 \cdot 1$ & 0.69 & $<0.0001$ & $7 \cdot 1$ \\
\hline Lutein + zeaxanthin $(\mu \mathrm{g} / \mathrm{d})$ & 0.56 & $<0.01$ & $7 \cdot 1$ & $0 \cdot 34$ & 0.074 & $7 \cdot 1$ & 0.35 & 0.065 & $10 \cdot 7$ \\
\hline Lycopene $(\mu \mathrm{g} / \mathrm{d})$ & 0.53 & $<0.01$ & $7 \cdot 1$ & 0.45 & $<0.05$ & $7 \cdot 1$ & 0.38 & $<0.05$ & $14 \cdot 3$ \\
\hline Flavonoids (mg/d) & $0 \cdot 89$ & $<0.0001$ & 0 & $0 \cdot 86$ & $<0.0001$ & 0 & 0.76 & $<0.0001$ & 0 \\
\hline Isoflavones (mg/d) & $0 \cdot 72$ & $<0.0001$ & $7 \cdot 1$ & $0 \cdot 73$ & $<0.0001$ & $4 \cdot 0$ & $0 \cdot 6$ & $<0.01$ & $7 \cdot 1$ \\
\hline Anthocyanins (mg/d) & 0.69 & $<0.001$ & 3.5 & 0.66 & $<0.001$ & 0 & 0.53 & $<0.01$ & $10 \cdot 7$ \\
\hline Flavan-3-ols (mg/d) & 0.91 & $<0.0001$ & 0 & $0 \cdot 89$ & $<0.0001$ & 0 & 0.77 & $<0.0001$ & $7 \cdot 1$ \\
\hline Flavanones (mg/d) & $0 \cdot 80$ & $<0.0001$ & $3 \cdot 5$ & $0 \cdot 75$ & $<0.0001$ & $4 \cdot 0$ & 0.55 & $<0.01$ & $10 \cdot 7$ \\
\hline Flavones (mg/d) & $0 \cdot 35$ & 0.065 & $10 \cdot 7$ & 0.39 & $<0.05$ & $10 \cdot 7$ & $0 \cdot 31$ & $0 \cdot 113$ & $10 \cdot 7$ \\
\hline Flavonols (mg/d) & $0 \cdot 82$ & $<0.0001$ & 0 & $0 \cdot 84$ & $<0.0001$ & 0 & $0 \cdot 65$ & $<0.001$ & $10 \cdot 7$ \\
\hline Proanthocyanidins (mg/d) & $0 \cdot 84$ & $<0.0001$ & 0 & $0 \cdot 82$ & $<0.0001$ & 0 & 0.69 & $<0.0001$ & 0 \\
\hline TAC (mg VCE/d) & $0 \cdot 82$ & $<0.0001$ & 0 & $0 \cdot 78$ & $<0.0001$ & 0 & $0 \cdot 78$ & $<0.0001$ & 0 \\
\hline
\end{tabular}

7dFR, $7 \mathrm{~d}$ food record; Ave FR, averaged four 7dFR, Ave FFQ, averaged four FFQ; $r$, Spearman correlation coefficient; \% Mis-C, percentage of misclassification; TAC, total antioxidant capacity; VCE, vitamin C equivalent. *Individual antioxidants and TAC were estimated from diet.

tPercentage of respondents categorized into the extreme opposite tertile.

Table 5 Spearman rank correlations between dietary antioxidants estimated from FFQ1 and FFQ4 in overweight and obese postmenopausal women who completed four sets of $7 \mathrm{dFR}$ and FFQ during 9 months ( $n$ 35)

\begin{tabular}{lcc}
\hline & \multicolumn{2}{c}{ FFQ1 v. FFQ4 (reliability) } \\
\cline { 2 - 3 } & \multicolumn{2}{c}{ Spearman correlation } \\
\cline { 2 - 3 } Nutrient & $r$ & $P$ value \\
\hline Diet vitamin E $(\mathrm{mg} / \mathrm{d})$ & 0.46 & $<0.01$ \\
Diet $\alpha$-tocopherol $(\mathrm{mg} / \mathrm{d})$ & 0.90 & $<0.0001$ \\
Diet $\gamma$-tocopherol $(\mathrm{mg} / \mathrm{d})$ & 0.24 & 0.161 \\
Diet vitamin C $(\mathrm{mg} / \mathrm{d})$ & 0.82 & $<0.0001$ \\
Diet carotenoids $(\mu \mathrm{g} / \mathrm{d})$ & 0.74 & $<0.0001$ \\
$\alpha$-Carotene $(\mu \mathrm{g} / \mathrm{d})$ & 0.91 & $<0.0001$ \\
$\beta$-Carotene $(\mu \mathrm{g} / \mathrm{d})$ & 0.49 & $<0.01$ \\
$\beta$-Cryptoxanthin $(\mu \mathrm{g} / \mathrm{d})$ & 0.31 & 0.071 \\
Lutein + zeaxanthin $(\mu \mathrm{g} / \mathrm{d})$ & 0.49 & $<0.01$ \\
Lycopene $(\mu \mathrm{g} / \mathrm{d})$ & 0.75 & $<0.0001$ \\
Diet flavonoids $(\mathrm{mg} / \mathrm{d})$ & 0.65 & $<0.0001$ \\
Isoflavones $(\mathrm{mg} / \mathrm{d})$ & 0.42 & $<0.05$ \\
Anthocyanins $(\mathrm{mg} / \mathrm{d})$ & 0.51 & $<0.01$ \\
Flavan-3-ols $(\mathrm{mg} / \mathrm{d})$ & 0.50 & $<0.01$ \\
Flavanones $(\mathrm{mg} / \mathrm{d})$ & 0.33 & 0.051 \\
Flavones $(\mathrm{mg} / \mathrm{d})$ & 0.70 & $<0.0001$ \\
Flavonols $(\mathrm{mg} / \mathrm{d})$ & 0.71 & $<0.0001$ \\
Diet proanthocyanidins $(\mathrm{mg} / \mathrm{d})$ & 0.78 & $<0.0001$ \\
Diet TAC $(\mathrm{mg} \mathrm{VCE} / \mathrm{d})$ & 0.63 & $<0.0001$ \\
\hline
\end{tabular}

7d FR, 7d food record; TAC, total antioxidant capacity; VCE, vitamin C equivalent.

have a greater daily variation than macronutrients ${ }^{(43,48)}$ and the corresponding food sources may be highly influenced by food availability. Therefore, to extend the period of dietary data collection using the FFQ, seasonal variability needs to be assessed using multiple FFQ collected every season during the reference period and validity also needs to be tested against multiple food records ${ }^{(38)}$. We did not find a time effect on dietary antioxidant intakes over 9 months using four sets of both 7dFR and FFQ. Analysis of food sources contributing to specific antioxidant intakes indicated consistent consumption of major food contributors that most affected the corresponding antioxidants (data not shown). A number of previous investigations documented differences in fruit and vegetable consumption across chronologic seasons and found that vegetables were generally consumed year-round whereas certain fruits were eaten primarily in certain seasons ${ }^{(49,50)}$. However, few studies have measured the seasonal variation of antioxidant intakes. We identified a study that used an FFQ to assess seasonal impact on micronutrient intakes in Ireland ${ }^{(51)}$. That study did not find any significant variation in micronutrient intake with respect to the month or the season. Nevertheless, convincing data have not been fully reported yet in this respect. In the present study, repeated 7dFR collected every season were used as a reference measure to compare long-term antioxidant intake against the FFQ and we found that this FFQ is capable of reflecting year-round antioxidant intake without being influenced by seasonal change. Moderate to high correlation coefficients of most antioxidants were observed between these two methods, which were within the ranges reported by previous studies ${ }^{(33-40,52)}$, and only $4 \%$ to $\sim 9 \%$ of participants were allocated into the completely opposite tertile. Taking into account desirable cut-off values suggested by Masson et al. ${ }^{(53)}$ (Spearman correlation coefficient above 0.5 ; less than $10 \%$ for misclassification), most nutrients coincided with these cut-off 
standards except for $\gamma$-tocopherol, certain carotenoids and flavones. On the whole, based on different statistical methods, it can be concluded that this 1-month FFQ can be used for estimating long-term comprehensive antioxidant intake in this population.

This FFQ has matured sufficiently to exhibit several strengths. First of all, to the authors' knowledge, this is the first FFQ developed to specifically investigate a complete antioxidant intake profile. Although previous studies have documented the assessment of antioxidant vitamins or certain flavonoids using an FFQ, no information is available in the literature about extensive estimations of individual antioxidant intake as well as dietary TAC. Second, validation of the FFQ in assessing antioxidant intake prudently considered the daily variation and seasonal change of antioxidant intake and the 'gold standard' reference. Third, multiple statistical approaches in the validity tests and comparisons with previous validation studies attested to the applicability and comprehensiveness of the FFQ tested. However, the interpretation of the present study is limited by a small sample size of overweight postmenopausal women from the north-east USA. The usefulness of this FFQ in this target group does not guarantee it would function well in other populations, especially with respect to the observed seasonal impact on antioxidant intake. As a result, the duration may be extended to 1 year and seasonal questions may be incorporated to improve the validity of the FFQ in different populations.

\section{Conclusions}

The new questionnaire generally provides a comprehensive antioxidant intake profile and reasonable rankings of selfreported dietary estimates through multiple statistical tools including correlation analysis, cross-classification and (more rigorously) biochemical surrogates. No significant seasonal variation of antioxidant intakes supports the applicability of this 1-month FFQ in capturing a usual dietary antioxidant pattern with respect to these overweight postmenopausal women. The validated comprehensiveness of this FFQ in assessing both short-term and long-term antioxidant intake may justify the use of this FFQ in associating dietary antioxidants with disease risks in this population.

\section{Acknowledgements}

Sources of funding: The present study was supported by the Donaghue Nutrition Research Program. Conflict of interest: All authors have no conflicts of interest to declare. Ethics: The study was approved by the Human Investigation Review Committees of the University of Connecticut Health Center and the University of Connecticut Institutional Review Board. Authors' contributions: O.K.C. developed the concept. O.K.C. and M.Y. prepared the preliminary data, developed the FFQ and designed the validation study. M.Y.,
Y.W. and C.G.D. acquired the data. S.G.L. analysed the plasma biomarkers. M.Y. conducted the statistical analysis and drafted the manuscript. S.I.K., M.L.F. and E.C. provided technical support and advice as members of the project steering group. All authors were involved in the data interpretation and manuscript preparation. Acknowledgements: The authors would like to thank all of the postmenopausal women who participated in the study and colleagues in the General Clinical Research Center of the University of Connecticut Health Center for their help.

\section{References}

1. Genkinger JM, Platz EA, Hoffman SC et al. (2004) Fruit, vegetable, and antioxidant intake and all-cause, cancer, and cardiovascular disease mortality in a communitydwelling population in Washington County, Maryland. Am J Epidemiol 160, 1223-1233.

2. Heidemann C, Schulze MB, Franco OH et al. (2008) Dietary patterns and risk of mortality from cardiovascular disease, cancer, and all causes in a prospective cohort of women. Circulation 118, 230-237.

3. Arai Y, Watanabe S, Kimira M et al. (2000) Dietary intakes of flavonols, flavones and isoflavones by Japanese women and the inverse correlation between quercetin intake and plasma LDL cholesterol concentration. J Nutr 130, 2243.

4. Knekt P, Kumpulainen J, Jarvinen R et al. (2002) Flavonoid intake and risk of chronic diseases. Am J Clin Nutr 76, $560-568$

5. Kris-Etherton PM, Hecker KD, Bonanome A et al. (2002) Bioactive compounds in foods: their role in the prevention of cardiovascular disease and cancer. Am JMed 113, Suppl. 9B, 71S-88S

6. Liu RH (2003) Protective role of phytochemicals in whole foods: implications for chronic disease prevention. Appl Biotechnol Food Sci Policy 1, 39-46.

7. Rimm EB, Katan MB, Ascherio A et al. (1996) Relation between intake of flavonoids and risk of coronary heart disease in male health professionals. Ann Intern Med 125, 384-389.

8. Sesso H, Gaziano J, Liu S et al. (2003) Flavonoid intake and the risk of cardiovascular disease in women. Am J Clin Nutr 77, 1400-1408.

9. Sesso H, Paffenbarger RJ, Oguma Y et al. (2003) Lack of association between tea and cardiovascular disease in college alumni. Int J Epidemiol 32, 527-533.

10. Satia JA, Waiters JL \& Galanko JA (2009) Validation of an antioxidant nutrient questionnaire in whites and African Americans. J Am Diet Assoc 109, 502-508.

11. Willett WC (1994) Future directions in the development of food-frequency questionnaires. Am J Clin Nutr 59, 1 Suppl., 171S-174S.

12. Yang M, Wang Y, Davis CG et al. (2012) Validation of an FFQ to assess short-term antioxidant intake against $30 \mathrm{~d}$ food records and plasma biomarkers. Public Health Nutr (Epublication ahead of print version).

13. Weyer C, Yudkin J, Stehouwer C et al. (2002) Humoral markers of inflammation and endothelial dysfunction in relation to adiposity and in vivo insulin action in Pima Indians. Atherosclerosis 161, 233-242.

14. Billington C, Epstein LH, Goodwin NJ et al. (2000) Overweight, obesity, and health risk. Arch Intern Med 160, 898-904.

15. Yang M, Chung S-J, Chung CE et al. (2011) Estimation of total antioxidant capacity from diet and supplements in US adults. Br J Nutr 105, 254-263.

16. National Center for Health Statistics (2002) National Health and Nutrition Examination Survey, 1999-2000 
Data Files. Hyattsville, MD: Centers for Disease Control and Prevention; available at http://www.cdc.gov/nchs/nhanes/ nhanes1999-2000/nhanes99_00.htm

17. National Center for Health Statistics (2004) National Health and Nutrition Examination Survey, 2001-2002 Data Files. Hyattsville, MD: Centers for Disease Control and Prevention; available at http://www.cdc.gov/nchs/data/nhanes/ nhanes_01_02/136_b_doc.pdf

18. Agricultural Research Service, US Department of Agriculture (2013) Database for the Flavonoid Content of Selected Foods. Beltsville, MD: USDA; available at http://www.ars. usda.gov/Services/docs.htm?docid $=6231$

19. Agricultural Research Service, US Department of Agriculture (2002) USDA-Iowa State University Database on the Isoflavone Content of Foods, Release 2.0. Beltsville, MD: USDA; available at http://www.ars.usda.gov/Services/docs. htm?docid $=6382$

20. Agricultural Research Service, US Department of Agriculture (2004) Database for the Proanthocyanidin Content of Selected Foods. Beltsville, MD: USDA; available at http:// www.ars.usda.gov/Services/docs.htm?docid $=5843$

21. Kim D-O, Chun OK, Kim YJ et al. (2003) Quantification of polyphenolics and their antioxidant capacity in fresh plums. J Agric Food Chem 51, 6509-6515.

22. Chun OK, Floegel A, Chung SJ et al. (2010) Estimation of antioxidant intakes from diet and supplements in US adults. J Nutr 140, 317-324.

23. Nutrition Coordinating Center, University of Minnesota (2010) Nutrition Data System for Research, Release 2010. Minneapolis, MN: NCC; available at http://www.ncc.umn. edu/products/ndsr.html

24. National Center for Health Statistics (2010) National Health and Nutrition Examination Survey, 2007-2008 Dietary Supplement Use. Hyattsville, MD: Centers for Disease Control and Prevention; available at http://www. cdc.gov/nchs/nhanes/nhanes2007-2008/DSDOC_E.htm

25. Ross MA (1994) Determination of ascorbic acid and uric acid in plasma by high-performance liquid chromatography. J Chromatogr B 657, 197-200.

26. Leonard SW, Bruno RS, Paterson E et al. (2003) 5-Nitro$\gamma$-tocopherol increases in human plasma exposed to cigarette smoke in vitro and in vivo. Free Radic Biol Med 35, 1560-1567.

27. Karppia J, Nurmia T, Olmedilla-Alonsob B et al. (2008) Simultaneous measurement of retinol, $\alpha$-tocopherol and six carotenoids in human plasma by using an isocratic reversedphase HPLC method. J Chromatogr B 867, 226-232.

28. van den Berg R, Haenen GRMM, van den Berg H et al. (1999) Applicability of an improved Trolox equivalent antioxidant capacity (TEAC) assay for evaluation of antioxidant capacity measurements of mixtures. Food Chem 66, 511-517.

29. Floegel A, Kim DO, Chung SJ et al. (2010) Development and validation of an algorithm to establish a total antioxidant capacity database of the US diet. Int $J$ Food Sci Nutr 61, 600-623.

30. Bland JM \& Altman DG (1986) Statistical-methods for assessing agreement between 2 methods of clinical measurement. Lancet 1, 307-310.

31. Cade J, Thompson R, Burley V et al. (2002) Development, validation and utilisation of food-frequency questionnaires - a review. Public Health Nutr 5, 567-587.

32. Hodge AM, Simpson JA, Fridman M et al. (2009) Evaluation of an FFQ for assessment of antioxidant intake using plasma biomarkers in an ethnically diverse population. Public Health Nutr 12, 2438-2447.

33. Andersen LF, Solvoll K, Johansson LR et al. (1999) Evaluation of a food frequency questionnaire with weighed records, fatty acids, and $\alpha$-tocopherol in adipose tissue and serum. Am J Epidemiol 150, 75-87.
34. Bautista LE, Herran OF \& Pryer JA (2005) Development and simulated validation of a food-frequency questionnaire for the Colombian population. Public Health Nutr 8, 181-188.

35. MacIntyre UE, Venter CS \& Vorster HH (2001) A culturesensitive quantitative food frequency questionnaire used in an African population: 1. Development and reproducibility. Public Health Nutr 4, 53-62.

36. Sauvaget C, Allen N, Hayashi M et al. (2002) Validation of a food frequency questionnaire in the Hiroshima/Nagasaki Life Span Study. J Epidemiol 12, 394-401.

37. Date C, Fukui M, Yamamoto A et al. (2005) Reproducibility and validity of a self-administered food frequency questionnaire used in the JACC study. J Epidemiol 15, Suppl. 1, S9-S23.

38. Henriquez-Sanchez P, Sanchez-Villegas A, Doreste-Alonso J et al. (2009) Dietary assessment methods for micronutrient intake: a systematic review on vitamins. Br J Nutr 102, Suppl. 1, S10-S37.

39. Lee MS, Pan WH, Liu KL et al. (2006) Reproducibility and validity of a Chinese food frequency questionnaire used in Taiwan. Asia Pac J Clin Nutr 15, 161-169.

40. Schroder H, Covas MI, Marrugat J et al. (2001) Use of a three-day estimated food record, a 72-hour recall and a food-frequency questionnaire for dietary assessment in a Mediterranean Spanish population. Clin Nutr 20, 429-437.

41. McNaughton SA, Marks GC, Gaffney P et al. (2005) Validation of a food-frequency questionnaire assessment of carotenoid and vitamin $\mathrm{E}$ intake using weighed food records and plasma biomarkers: the method of triads model. Eur J Clin Nutr 59, 211-218.

42. Satia JA, Watters JL \& Galanko JA (2009) Validation of an antioxidant nutrient questionnaire in whites and African Americans. J Am Diet Assoc 109, 502-508.

43. Willett W (2002) Nutritional Epidemiology. New York: Oxford University Press.

44. Labonte ME, Cyr A, Baril-Gravel L et al. (2012) Validity and reproducibility of a web-based, self-administered food frequency questionnaire. Eur J Clin Nutr 66, 166-173.

45. Dixon LB, Subar AF, Wideroff L et al. (2006) Carotenoid and tocopherol estimates from the NCI diet history questionnaire are valid compared with multiple recalls and serum biomarkers. J Nutr 136, 3054-3061.

46. Pellegrini N, Salvatore S, Valtuena S et al. (2007) Development and validation of a food frequency questionnaire for the assessment of dietary total antioxidant capacity. $J$ Nutr 137, 93-98.

47. Ocke MC, Bueno-de-Mesquita HB, Goddijn HE et al. (1997) The Dutch EPIC food frequency questionnaire. I. Description of the questionnaire, and relative validity and reproducibility for food groups. Int J Epidemiol 26, Suppl. 1, S37-S48.

48. Davis CG (2010) Estimation of the number of days required to determine usual antioxidant intakes and assessment of the prevalence of nutrient inadequacy among college students. Master Thesis, University of Connecticut.

49. Locke E, Coronado GD, Thompson B et al. (2009) Seasonal variation in fruit and vegetable consumption in a rural agricultural community. J Am Diet Assoc 109, 45-51.

50. Ziegler RG, Wilcox HB, Mason TJ et al. (1987) Seasonalvariation in intake of carotenoids and vegetables and fruits among white men in New Jersey. Am J Clin Nutr 45, 107-114.

51. O'Connell ED, Nolan JM, Stack J et al. (2008) Diet and risk factors for age-related maculopathy. Am J Clin Nutr 87, 712-722.

52. Block G, Woods M, Potosky A et al. (1990) Validation of a self-administered diet history questionnaire using multiple diet records. J Clin Epidemiol 43, 1327-1335.

53. Masson LF, McNeill G, Tomany JO et al. (2003) Statistical approaches for assessing the relative validity of a foodfrequency questionnaire: use of correlation coefficients and the kappa statistic. Public Health Nutr 6, 313-321. 